Physics

Weights \& Measures fields

Year 1999

\title{
Range imaging system with multiplexed structured light by direct space encoding
}

\author{
Mitsuru Baba \\ Tadataka Konishi \\ Okayama University \\ Okayama University
}

This paper is posted at eScholarship@OUDIR : Okayama University Digital Information Repository.

http://escholarship.lib.okayama-u.ac.jp/weights_and_measures/3 


\title{
Range Imaging System with Multiplexed Structured Light by Direct Space Encoding
}

\author{
Mitsuru Baba and Tadataka Konishi \\ Faculty of Engineering, Okayama Univrsity \\ 1-1 Naka 3-chome, Okayama, 700-0082, Japan \\ baba@sdc.it.okayama-u.ac.jp
}

\begin{abstract}
Since practical multiplexed structured light systems currently available use plural light patterns or different illuraination conditions to ensure a high reliability, their fast performance is impaired. This paper describes a fast, highly reliable range imaging system with a multiplexed structured light system that uses a direct space encoding approach while using only a single light pattern. Unlike a conventional encoding approach, the proposed approach is unique in that it encodes object space through the use of a special optical system which consists of field stops, plural lenses, and shield masks, rather than a light pattern. The theoretical considerations and experimental results demonsirate that the proposed approach is effective for a highly reliable, fast, acturate range imaging system.
\end{abstract}

\section{Introduction}

Most practical range imaging systems at the present time employ triangulation-based structured light methods[1]. The most popular form of these structured merhods uses the projection of a single narrow light stripe on a scene that consequently scans the entire area, because th is method has the greatest potential for accurate, high-range resolution and is highly reliable. However, projecting a large number of light stripes in a denser construction is a fairly slow process[2].

The range sensing process can be speeded up if multiple light stripes (rather than one single stripe) are employed[3][5]. These multiplexed structured light approaches require stripe identification. The multiplexed structured light approaches currently in use typically identify the light patiern by certain markings. Since the markings occupy some space that must remain conjected in the image of the illuminated scene, when using these approaches it is generally difficult to completely eliminate ambiguity in the identification of the light pattern. In addition, using this approach inevitably produces some errors in detecting of the light projection angle, and thus there arises a position error corresponding to each light stripe. Therefore, the typical multiplexed structured light stripe system has lower reliability and less range accuracy than does a single light stripe system.

Recently, several practical multiplexed structured light stripe approaches have been suggested in order to alleviate these problems, including time multiplexing approaches using gray codes[6] and an adaptive color structured light approach[7]. However, these approaches require the acquisition of several image in order to ensure high reliability and good accuracy, and therefore they have suffered from one inherent drawback, namely, speed. If a practical multiplexed structured light system using only a single light pattern can be realized, the above drawback can be overcome easily.

In this paper, we describe a highly reliable, fast, accurate range imaging system based on a novel multiplexed structured light approach that uses only a single light pattern. The proposed approach is constructed using a specially devised optical system which divides the measuring space into the same number of areas as that of the projected light stripes. Unlike the conventional encoding approaches, our approach encodes the measuring space not indirectly by the light pattern but directly by the optical system, and thus the system meets the needs for high-speed measurements, high reliability and high range accuracy.

\section{Multiplexed structured light approach}

\subsection{Previlous approaches}

Systems using classical multiplexed structured light approaches project a light pattern to encode measuring space as shown in Fig. 1. The light pattern might, for example, include multiple stripes of the same color, color-coded stripes, a grid, and a circle[1]. Any of these light patterns is associated with a given set of projecting points, and the depth can be calculated by triangulation. However, simultaneous projection of the light pattern to an entire scene can result in ambiguities, which in turn can cause significant errors in the correspondence between a light pattern and the associated projecting points. 


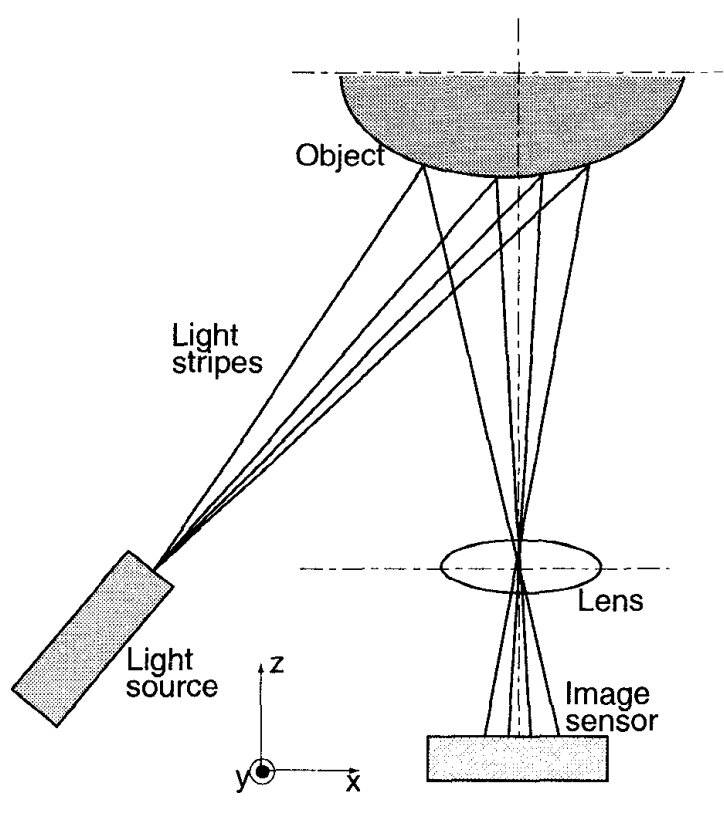

Fig.1 Conventional multiplexed structurd light approach

The typical approaches aimed at solving this problem are the time multiplexing approach using gray codes and the adaptive color structured light approach. The time multiplexing approach, which encodes the multiple stripes by assigning gray codes to the light pattern and sequentially projects several different gray-coded patterns, has been a robust and widely applied structured light system. This method can acquire the equivalent information to that obtained with $2^{n}$ by $n$ project patterns. Although it determines the range image by detecting the edge of these patterns, the ambiguity in the extraction of the code corresponding to the boundary of the projected pattern is limited to plus-or-minus 1 LSB (least significant bit). An alternative approach is the adaptive color structured light approach, which automatically adapts the number and form of the projection patterns suitable for the characteristics of the scene. Typical color-coded light approaches are practical only in a measuring environment that reflects all wavelengths of light. However, this approach also uses several images, which are acquired by different illumination conditions, and thus it reduces the constraint of the measuring environment.

Both of these approaches require several projections of the light stripe patterns, which results in increased sampling time.

\subsection{Proposed approach}

Fig. 2 shows the schematic diagram of our proposed multiplexed structured light approach, which consists of field stops, lenses, shield masks, and image sensors. In this ap-
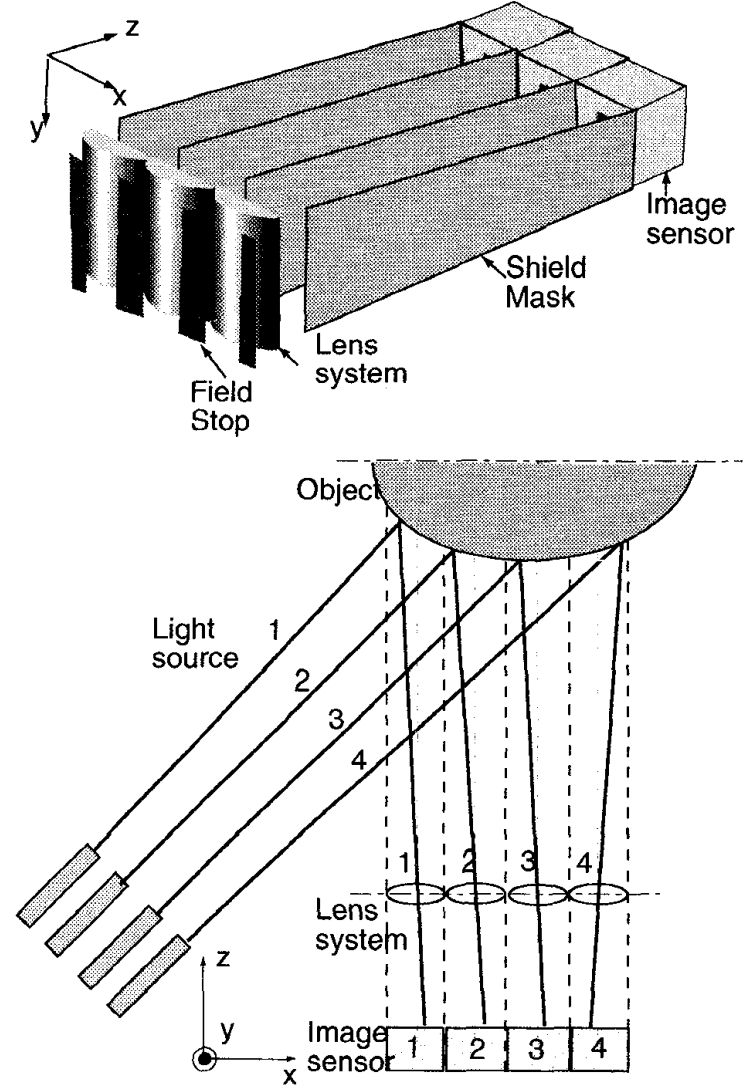

Fig.2 Proposed multiplexed structured light approach

proach, multiple light stripes are projected simultaneously within an arrangement of lenses and image sensors equal in number to the number of lights projected. As shown in Fig. 2 , we adjust the arrangement of the components of the optical system in such a manner that the reflected light from the object in the $i$-th encoded region reaches only the $i$-th image sensor via only the $i$-th lens. As a result, the proposed method can encode the measuring space without any ambiguity. The conventional encoding approach indirectly encodes a measuring space by embedding identifying information in the light pattern. In contrast, our encoding approach directly encodes the measuring space using the special optical system rather than a light pattern. This has two advantages: (1) highly reliable characteristics in a single light projection method, and (2) fast performance in a multiplexed light stripes projection approach. 


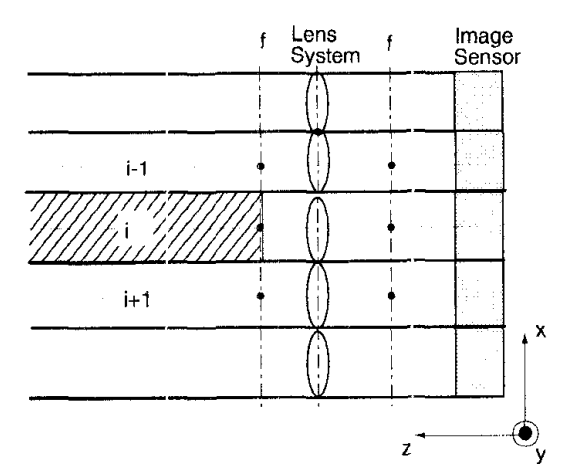

Fig.3 Proposed space encoding principle

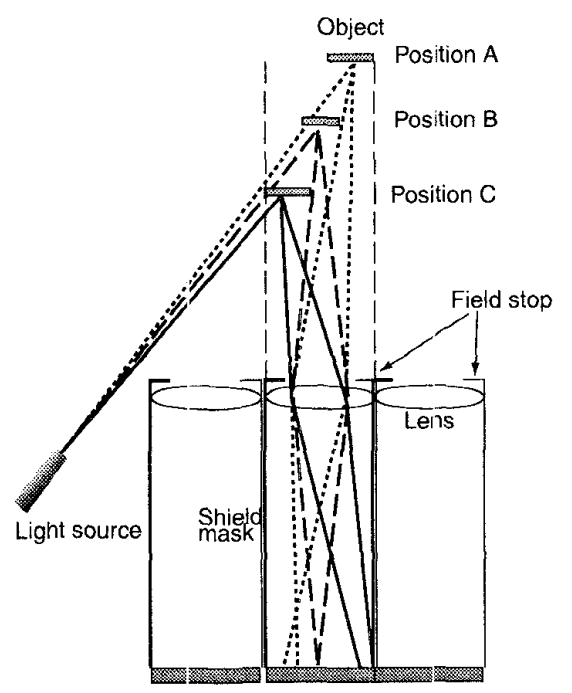

Fig.4 Theoretical result of the ray tracing by the proposed approach I

\section{Theoretical analysis of the proposed approach}

Taking Fig. 3 as an example, the proposed approach needs to meet the following two optical requirements: (1) In the $i$ th region, all point sources of light that exist in the shaded portion must reach the only image sensor in that region. (2) In the $(i-1)$-th and $(i+1)$-th regions adjacent to the $i$-th region, none of the point sources of light must reach the image sensor of the $i$-th region. We confirmed the technical feasibility of the proposed approach by ray tracing with an analysis of geometrical optics[3].

Fig. 4 shows an example of the theoretical results of ray tracing on the light reflected from the three objects' positions, while focusing attention on one region. In this figure, the focal length is $20 \mathrm{~mm}$ of the lens, and the distance between object and lens is $30 \mathrm{~mm}$ (position C), $40 \mathrm{~mm}$ (posi-

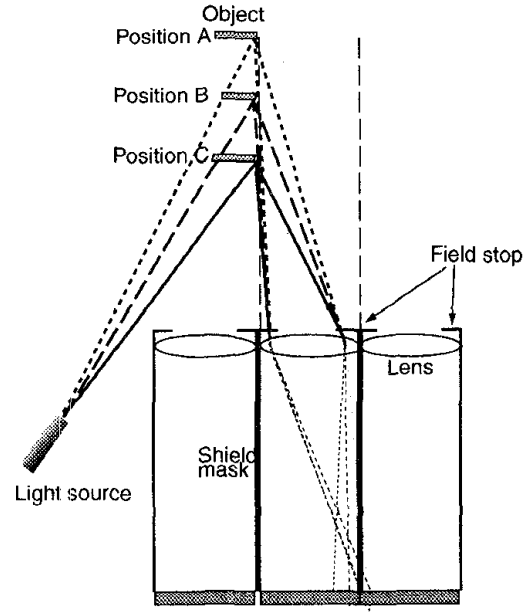

Fig.5 Theoretical result of the ray tracing by the proposed approach II

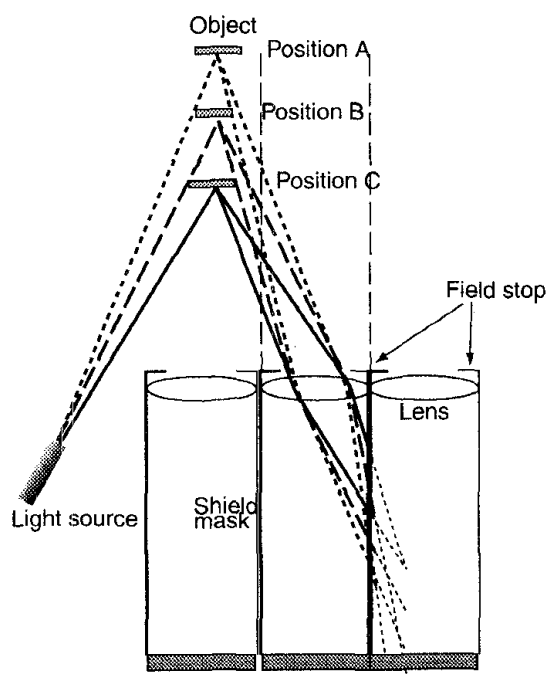

Fig.6 Theoretical result of the ray tracing by the proposed approach III

tion $\mathrm{B}$ ), and $50 \mathrm{~mm}$ (position A), respectively. The in-focus distances of each object's position are $33 \mathrm{~mm}, 40 \mathrm{~mm}$, and $62 \mathrm{~mm}$, respectively. In the case of this position of the image sensor, position $\mathrm{B}$ is in focus, and position $\mathrm{A}$ and position $\mathrm{C}$ are unfocused, but the centers of the blur of both cases are within the region in question. This analysis demonstrates that three lights reflected from objects located in different positions of the same measuring region can focus into the image sensor of the region in question.

On the other hand, Fig. 5 and Fig. 6 show the case of the light reflected from the object out of the region in question. The reflected points of Fig. 5 are in close vicinity to the border of the region, and the reflected points of Fig. 6 are far 
from the border. From these figures, it can be seen that all the light does not reach the image sensor in a given region. This figure shows that the field stops prevent the light of the adjacent region (for example, the light reflected from position $A$ and $B$ in Fig.5) from invading the region, and the shield masks prevent adjacent light through the lens (for example, the light reflected from positions C in Fig.5 and all points in Fig.6) from invading another adjacent region.

Like most ordinary range imaging methods, the range resolution of our approach mainly depends on the measurement resolution of the image sensor used for the system. On the other hand, with this approach spatial resolution is limited to the interval between adjacent stripes, and in such a case, the depth of field is given by $1 / \tan \gamma$, where $\gamma$ is the projection angle. Therefore, the depth of field decreases when spacial resolution is high. Unlike the case with ordinary multiplexed structured light approaches, the proposed approach does not need the decoding time of the structured light. As a result, the speed of image data acquisition depends only on the dwell time of the image sensor used.

\section{The system implementation}

\subsection{Optical system implementation}

We have designed and built a prototype range imaging system that gives a concrete form to our idea on space encoding. Fig. 7 illustrates the geometry of the system and the parameters employed. The system consists of a light source device, a lens system, a image sensor, a signal processing circuit, and a computer for measurement.

The light source device uses semiconductor laser diodes or a slide projector. For simplicity, we encoded the measuring space by dividing it into six regions, and we simultaneously projected six light stripes onto the scene. There were two lens systems, labeled lens system 1 and lens system 2 . Lens system 1 has one cylindrical lens, which is set in a

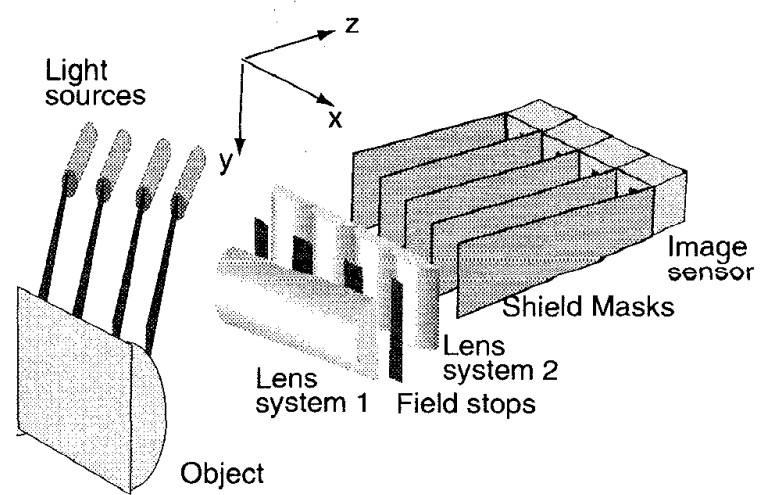

Fig.7 Prototype range imaging system horizontal direction, and it is used to determine a field of view in the vertical direction. Its focal length is $40 \mathrm{~mm}$, its width is $60 \mathrm{~mm}$, and its length is $90 \mathrm{~mm}$. Lens system 2 consists of 6 cylindrical lenses set in a vertical direction; it is used to divide the measuring range. An image sensor and the two lens systems are aligned on the $\mathrm{z}$-axis. The distance between the image sensor and object ranges from $190 \mathrm{~mm}$ to $210 \mathrm{~mm}$. The projection angle is 45 degrees relative to the $\mathrm{x}$-axis.

\subsection{Image sensor implementation}

We devised a new high-speed image sensor in order to make good use of the efficiency of the approach. Since our approach simultaneously projects multiple light stripes onto the scene, the image sensor must receive the light stripes simultaneously. Although range imaging systems normally used position-sensitive detector (PSD) [9] and chargecoupled device (CCD), these sensors are unable to simultaneously meet the requirements of our approach. The PSD can detect the position of a point at high speed, but it cannot in principle receive plural light stripes. In contrast, the CCD has the ability to detect plural points simultaneously; however, it is much slower than the PSD due to the scanning time needed.

The proposed image sensor is one of a linear array type and consists of scores of sensor array units that in turn comprise a small number of photovoltaic sensors, as shown in Fig. 8. The image sensor consists of $6 \times 12$ sensor array units composed of five photodiodes. The area of the received light of the sensor is $35 \mathrm{~mm} \times 32.5 \mathrm{~mm}$.

The image sensor detects the actual peak position using only an analog circuit. Fig. 9 illustrates the circuitry for detection of the peak position. Essential components in this circuitry include a current-voltage converting unit, an adding unit, an integrating unit, and a dividing unit. When the

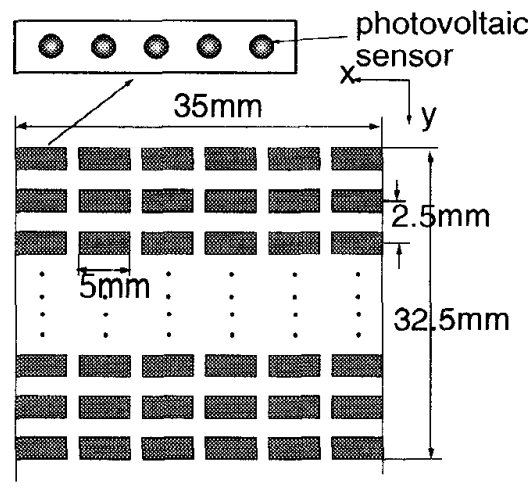

Fig.8 Proposed high-speed image sensor 


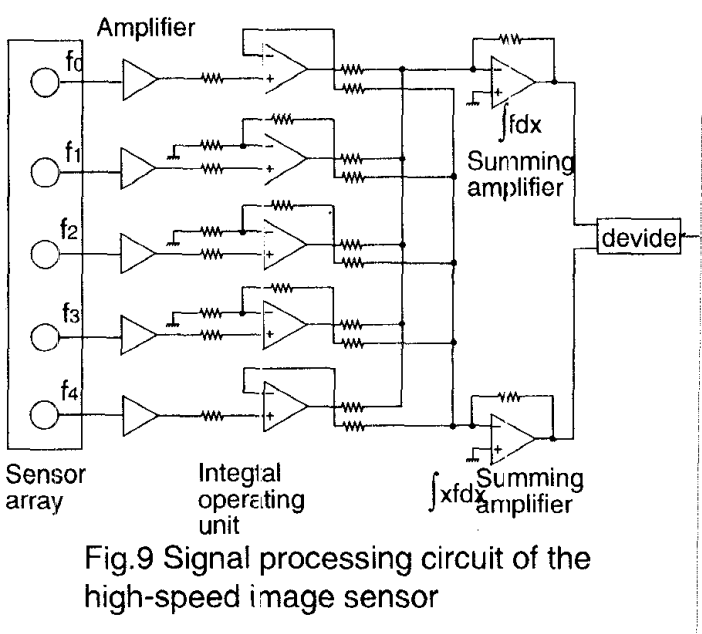

light stripes are input to the sensor array, the circuitry converts the current that is output from the photovoltaic to voltage. The adding unit and integrating unit output $F$ and $P$, respectively. $F$ and $P$ are expressed by the following equations:

$$
\begin{aligned}
X & =P / F \\
F & =\int f_{i} d x \\
& =\frac{3}{8} h\left(0.6 f_{0}+1.8 f_{1}+1.8 f_{2}+1.4 f_{3}+0.8 f_{4}\right)
\end{aligned}
$$

where $x_{i}$ is the position in the $x$-coordinate direction of the $\mathrm{i}$ th photovoltaic sensor from the left, and fi is the output of the $\mathrm{i}$-th photovoltaic sensor. Therefore, the actual position $\mathrm{x}$ of the light stripe is expressed by equation (3) as follows:

$$
\begin{aligned}
P & =\int x_{i} f_{i} d x \\
& =\frac{3}{8} h\left(0.3 f_{0}+2.7 f_{1}+4.5 f_{2}+4.9 f_{3}+3.6 f_{4}\right) \\
h & =\frac{x_{4}-x_{0}}{5}
\end{aligned}
$$

The circuitry actualizes the numerical integrating process by adjusting the gain of each operational amplifier. Also, the dividing unit outputs an actual peak position $(X)$ of the light stripe.

We measured the response time by inputting a high-frequency pulse in the image sensor and by checking whether or not the output of the sensor follows the input pulse. The sensor has a response of about 5 microseconds (this corresponds to a sampling irequency of $200 \mathrm{kHz}$ ), which is equivalent to the response time of a PSD.

\section{Experiment and discussion}

\subsection{Experimental verification of the encoding approach}

Fig. 10 shows the received distribution of the light stripes. This experiment divides the measuring space into five regions and projects only one light stripe for each region. In this experiment, the light stripes are projected on each region in sequence to verify light stripe identification. As a result, since all light stripes are projected onto the corresponding region, it is shown that the proposed method can unerringly encode the multiple light stripes.

\subsection{Shape measurement results}

Fig. 11 shows the measurement result for a object. In the case of this experiment, we divided the measuring space into six regions and moved the lens system and image sensor in such a manner as to be able to measure the entire object. The maximum absolute measuring error is within $0.8 \mathrm{~mm}$. The

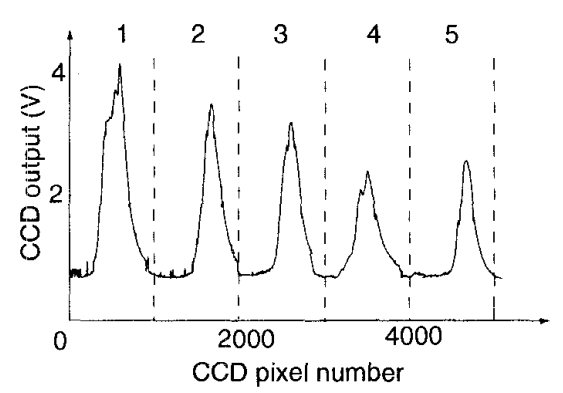

Fig.10 Measurement result of the distribution of the received light stripe

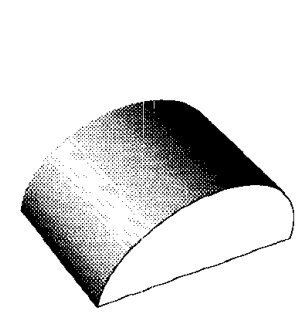

(a) Object

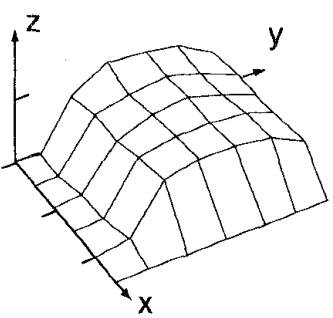

(b) Measurement result
Fig.11 3D display of measurement results I 


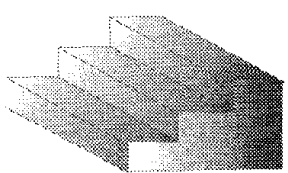

(a) Object

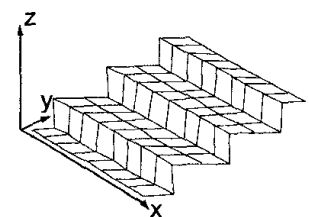

(b) Measurement result
Fig.12 3D display of measurement results II

Table 1 Perfoemance of the prototype system

\begin{tabular}{ll}
\hline Paremeter & Performance \\
\hline Standoff & $100-120 \mathrm{~mm}$ \\
Field of view & $30 \times 30 \mathrm{~mm}$ \\
Depth of field & $5 \mathrm{~mm}$ \\
Range accurcy & $0.8 \mathrm{~mm}$ \\
Data acquisition time & $6 \mu \mathrm{s} / \mathrm{point}$ \\
\hline
\end{tabular}

sampling time per one point is 5 microseconds. In this experiment, one attempt results in 72 measuring points, and it takes a total of about $0.5 \mathrm{~ms}$ to complete the experiment. Fig. 12 shows the measurement result for other objecr. System performance is summarized in Table 1.

\subsection{Major Practical Applications of the Proposed Approach}

The uniqueness of our approach is that it meets the needs for high speed, reliability, and accuracy. This is accomplished by the following new aspects of the proposed approach. Our method belongs to a type of multiplexed striped light system in the encoding process that has high-speed characteristics. However, the decoding process belongs to a type of single stripe light system, which features high reliability of identification of light and high range accuracy.

The especially effective applications of the proposed approach are (1) the high-speed measurement of an object with small depth and (2) fast three-dimensional shape recogni- tion. In order to further improve our system, we need to overcome several problems. One is that since the spatial resolution of the approach is limited by the width of the lens that encodes the measuring space, the resolution of the present system is low. Therefore, in order to improve spatial resolution, the present system needs to incorporate a light stripe scanning mechanism using a higher speed.

\section{Conclusions}

We presented a range imaging system with a multiplexed structured light approach that is highly reliable while using only one light stripe pattern. This approach allows us to encode object space directly by using field stops, multiple lenses, and shield masks. Analysis of the general optics of the proposed approach by ray tracing showed that the approach can unerringly encode object space using only one light stripe pattern. The theoretical considerations and experimental results demonstrate that the proposed approach is effective for a highly reliable, fast, accurate range imaging system.

\section{References}

[1] L. Jorge and C.Sanz, Advances in Machine Vision", Springer, 1989

[2] T. Kanade, A.Gruss, and L.R. Carley, A Very Fast VLSI Rangefinder", Proc. International Conference Robotics and Automation, pp1322-1329, 1991

[3] G.B.Porter and J.L...Mundy, A noncontact profile sensing system for visual inspection", In Proc. Workshop on Industrial Applications of Machine Vision, pp1 19-pp129

[4] J.L..Mundy and G.B.Porter, A three-dimensional sensor based on structured light. In Three-dimAensional mexhine vision, Kluwer Academic

[5] J.Jalkio, R.Kim and S.Case,"3D inspection using multi-stripe structured light", Opt.Engrg, pp964-974,1985,

[6]K. Sato and S. Inokuchi, "Three-Demensional Surface Measurement by Space Encoding Range Imaging", J.Robotic Systems, vol.2 pp27-39, 1985 .

[7]D.Caspi, N.Kiyati and J.Shamir,'Range Imaging with Adaptive Color Structured Light", IEEE Trans. Pattern and Machine Intelligence, vol. 20, pp470-480 1998.

[8] M.Bom and E. Wolf,Principles of Optics", Pergamon Press, 1970 